\title{
Synergistic activation of thermogenic adipocytes by a combination of PPARY activation, SMAD3 inhibition and adrenergic receptor activation ameliorates metabolic abnormalities in rodents
}

\author{
Tomohiro Matsumoto $^{1} \cdot$ Satomi Kiuchi $^{1} \cdot$ Takatoshi Murase $^{1}$ (D) \\ Received: 1 February 2019 / Accepted: 16 May 2019 / Published online: 17 July 2019 \\ (C) Springer-Verlag GmbH Germany, part of Springer Nature 2019
}

\begin{abstract}
Aims/hypothesis To treat obesity and related diseases, considerable effort has gone into developing strategies to convert white adipocytes into thermogenic brown-like adipocytes ('browning'). The purpose of this study was to identify the most efficient signal control for browning.

Methods To identify the most efficient signal control for browning, we examined rat stromal vascular fraction cells. In addition, physiological changes consequent to signal control were examined in vivo using lean and diet-induced obese (DIO) C57BL/6J mice.

Results Combined treatment with the peroxisome proliferator-activated receptor $\gamma$ (PPAR $\gamma$ ) agonist rosiglitazone, the SMAD3 inhibitor SIS3 and the adrenergic receptor agonist noradrenaline (norepinephrine) synergistically induced Ucp $1, F g f 21$ and Cited 1 expression, triggering brown adipogenesis. Synergistic induction of $U c p 1$ by the three agents was negatively regulated by forkhead box O (FOXO)3 via the inhibition of PPAR $\gamma$-dependent gene transcription. Moreover, the administration of rosiglitazone, SIS3 and the selective $\beta 3$ adrenergic receptor agonist CL316,243 to DIO mice reduced the amount of body-fat deposits (body weight from day 0 to $14,12.3 \%$ reduction), concomitant with morphological changes in white adipose tissue, an increase in mitochondrial biosynthesis and a marked induction of uncoupling protein 1 (UCP1). Furthermore, administration of the three agents significantly increased serum adiponectin levels (mean $65.56 \mu \mathrm{g} / \mathrm{ml}$ with the three agents vs $20.79 \mu \mathrm{g} / \mathrm{ml} \mathrm{in}$ control mice, $p<0.05$ ) and improved glucose and lipid tolerance.

Conclusions/interpretation These results suggest that the combined regulation of PPAR $\gamma$, SMAD and the adrenergic receptor signalling pathway synergistically induces brown adipogenesis and may serve as an effective strategy to treat obesity and related diseases, including type 2 diabetes.
\end{abstract}

Keywords Adipocytes · Browning · FOXO3 - Obesity · PPAR $\gamma$ agonist

\section{Abbreviations}

BAT Brown adipose tissue

CtBP C-terminal-binding protein

DIO Diet-induced obese

Tomohiro Matsumoto and Satomi Kiuchi contributed equally to this study.

Electronic supplementary material The online version of this article (https://doi.org/10.1007/s00125-019-4938-6) contains peer-reviewed but unedited supplementary material, which is available to authorised users.

Takatoshi Murase

murase.takatoshi@kao.com

Biological Science Laboratories, Kao Corporation, 2606 Akabane, Ichikai-machi, Haga-gun, Tochigi 321-3497, Japan
FOXO Forkhead box $\mathrm{O}$

iWAT Inguinal white adipose tissue

MYF $5 \quad$ Myogenic factor 5

nTZDpa Non-thiazolidinedione partial agonist of peroxisome proliferator-activated receptor $\gamma$

PGC1 $\alpha \quad$ Peroxisome proliferator-activated receptor $\gamma$ coactivator $1 \alpha$

PPAR $\gamma \quad$ Peroxisome proliferator-activated receptor $\gamma$

PRDM16 PRD1-BF1-RIZ1 homologous domain containing 16

siRNA Small interfering RNA

SIS3 Specific inhibitor of SMAD3

SVF Stromal vascular fraction

TZD Thiazolidinedione

UCP1 Uncoupling protein 1 


\section{Research in context}

\section{What is already known about this subject?}

- Browning of adipocytes has been suggested as a potentially effective treatment for obesity and type 2 diabetes

- Peroxisome proliferator-activated receptor $\gamma$ (PPAR $\gamma$ ), SMAD3 and adrenergic receptor signals regulate browning

- Multiple transcription factors, including PPAR $\gamma$ and cofactors, contribute to transcription of uncoupling protein 1 (UCP1), which dissipates energy as heat

\section{What is the key question?}

- What are the most promising adipocyte-browning signal combinations for diabetes treatment and what are their regulatory molecules?

\section{What are the new findings?}

- This study reveals a method of inducing highly efficient browning by controlling multiple transcription factors/cofactors in adipose tissue in vivo and in vitro

- High-efficiency browning with PPAR $\gamma$, SMAD3 and adrenergic receptor signals reduced metabolic abnormalities in high-fat-diet-induced obese mice

\section{How might this impact on clinical practice in the foreseeable future?}

- The results of this study present the possibility of developing new concomitant medicines for the treatment of type 2 diabetes

\section{Introduction}

Obesity is closely related to the onset of lifestyle-related diseases such as diabetes, hypertension and cardiovascular disease, and the increase in the number of individuals affected by these diseases because of changes in dietary habits and lifestyles has become a major social problem [1]. A potentially effective strategy to prevent or treat obesity is to increase energy expenditure through uncoupling protein 1 (UCP1) by converting white adipocytes into brown-like adipocytes ('browning'). Brown adipocytes are characterised by abundant expression of UCP1, and contain multilocular lipid droplets and many mitochondria. UCP1 decreases the proton gradient between mitochondrial membranes and dissipates energy as heat by uncoupling respiration from ATP synthesis [2, 3]. Rodent-based studies have demonstrated that interscapular brown adipose tissue (BAT) is crucial not only for cold tolerance, but also for maintaining whole-body energy homeostasis [4-6]. Although it had been thought that BAT diminishes with age and is less functional in adult humans [7], recent studies have shown that active brown adipocytes are present in adults $[8,9]$ and play a role in cold-induced thermogenesis. However, decreased activity of adipocytes associated with ageing has also been observed [10-12]. Thus, brown adipocytes have attracted attention as a therapeutic target for obesity and diabetes.
Brown adipocytes are classified into two types based on their differentiation lineage. Brown adipocytes in human infants and rodents are termed 'classical brown adipocytes' and are differentiated from myogenic factor 5 (MYF5)-positive precursor cells. In contrast, those appearing in white adipose tissue when bodies are exposed to a cold environment or adrenergic receptor agonists are termed 'beige adipocytes' and are derived from MYF5-negative precursor cells [13, 14]. Gene expression profiling has revealed that brown adipocytes present in adult humans are mainly composed of beige adipocytes $[13,15]$. Moreover, in the last decade, molecules that regulate the differentiation and activity of brown adipocytes have been partly clarified [14]. The central regulator of these processes is peroxisome proliferator-activated receptor $\gamma$ (PPAR $\gamma$ ), with thiazolidinediones (TZDs) acting as a ligand for PPAR $\gamma$ to induce browning of white adipose tissue following their chronic administration in mammals or application to cultured adipocyte precursor cells $[16,17]$. Another master regulator of browning, PRD1-BF1-RIZ1 homologous domain containing 16 (PRDM16), has been shown to suppress white adipocyte-selective gene expression by interacting with C-terminal-binding protein (CtBP)-1 and CtBP-2, which upon binding with PPAR $\gamma$ coactivator $1 \alpha(\mathrm{PGC} 1 \alpha)$ displaces CtBPs and induces brown adipocyte-selective gene expression, such as that of PGC1 $\alpha$ itself [18]. In addition, TGF- $\beta$ family molecules regulate the differentiation lineage of 
adipocytes via their receptors and downstream SMAD signalling $[19,20]$. Among these, the TGF-SMAD3 axis reportedly inhibits brown adipogenesis by suppressing PGC1 $\alpha$ expression [21]. Thus, the differentiation lineage of adipocytes is controlled by numerous transcription factors [22].

Recently, the effective control of browning has been proposed as a new approach for treating obesity and diabetes; therefore, numerous efforts have been made to explore the inducers of this process [23]. Although TZDs are known to induce thermogenic gene expression in cultured cells and mammals $[16,17]$, it is difficult to obtain a marked antiobesity effect using TZDs alone because of their unwanted side effects [24]. Thus, effective means of regulating browning for the purposes of anti-obesity and diabetes treatment have not yet been established. In the present study, we investigated intracellular signals that could efficiently induce browning of rat stromal vascular fraction (SVF) cells. Furthermore, we examined the efficacy of agents that regulate browningrelated signals in obesity and diabetes in mice.

\section{Methods}

\section{Preparation of SVF cells}

Minced inguinal adipose tissues from male Wistar rats (810 weeks old, Japan SLC, Shizuoka, Japan) were incubated in collagenase buffer $(0.05 \%$ collagenase, $4 \%$ BSA in Hanks' buffer) for $15 \mathrm{~min}$ at $37^{\circ} \mathrm{C}$. After adding the culture medium (DMEM supplemented with $10 \%$ FBS), the cell suspension was passed through $100 \mu \mathrm{m}$ nylon mesh and centrifuged for $5 \mathrm{~min}$ at $200 \mathrm{~g}$. The upper layer of the suspension was removed, and the pellet was resuspended in culture medium and used as SVF. All animal experiments were approved by the Animal Care Committee of Kao Corporation and performed in accordance with the committee's guidelines.

\section{Cell experiments}

SVF cells were cultured in DMEM $(+10 \%$ FBS $)$ on type I collagen-coated plates for 1 day ( $\sim 80 \%$ confluence). Cells were then exposed to the PPAR $\gamma$ agonist rosiglitazone (Wako Pure Chemical, Osaka, Japan) and/or the SMAD3 inhibitor SIS3 (specific inhibitor of SMAD3; Sigma-Aldrich, St Louis, MO, USA) for the indicated time period, followed by addition of the adrenergic receptor agonist noradrenaline (norepinephrine) (Wako) for $2 \mathrm{~h}$. After washing the cells with PBS, cells were used for further analyses.

\section{RNA isolation and real-time PCR}

Total RNA was isolated from cultured cells or adipose tissues using QIAzol and the RNeasy Mini Kit (Qiagen, Venlo, the
Netherlands). Isolated RNA was reverse-transcribed using the High-Capacity RNA-to-cDNA Kit (Thermo Fisher Scientific, Waltham, MA, USA) and real-time PCR was performed with gene-specific TaqMan probes (Thermo Fisher Scientific, see electronic supplementary material [ESM] Table 1) using a 7500 Fast Real-Time PCR System (Thermo Fisher Scientific). The expression of each gene was normalised to that of Rplp0.

\section{Western blot analysis}

Adipose tissues were homogenised in ice-cold RIPA buffer (Sigma-Aldrich) using a motor-driven pestle in a microcentrifuge tube. Cells were lysed in $1 \%$ SDS containing buffer. Supernatants were denatured at $95^{\circ} \mathrm{C}$ in SDS sample buffer. Equal amounts of protein were subjected to SDSPAGE, followed by electroblotting onto a PVDF membrane. Lysates were quantified using BCA Protein Assay Kit (Thermo Fisher Scientific). Blotted membranes were probed with each primary antibody $\left(1: 1000,4^{\circ} \mathrm{C}, 16 \mathrm{~h}\right.$; ESM Table 2$)$, followed by a secondary antibody (1:1000) using an immunoreaction enhancer solution (Can Get Signal; TOYOBO, Osaka, Japan). Blots were visualised using the ECL prime western blotting detection system (GE Healthcare, Marlborough, MA, USA) and ImageQuant LAS 4000 (Fujifilm, Tokyo, Japan).

\section{Immunohistochemistry}

Adipose tissues were dissected and fixed in $10 \%$ formaldehyde at room temperature and embedded in paraffin. Paraffin sections were mounted on slides, deparaffinised and probed with an anti-UCP1 antibody (no. 23841; Abcam, Cambridge, UK). Visualisation was performed using the anti-rabbit EnVision+ System-HRP (Agilent Technologies, Santa Clara, CA, USA) and the Liquid DAB Substrate-Chromogen System (Agilent Technologies).

\section{Electron microscopy}

Adipose tissues were fixed with $2 \%$ paraformaldehyde and $2 \%$ glutaraldehyde in $0.1 \mathrm{~mol} / 1$ cacodylate buffer, $\mathrm{pH} 7.4$, at $4^{\circ} \mathrm{C}$ overnight, then subjected to post-fixation with $2 \%$ osmium tetroxide at $4^{\circ} \mathrm{C}$ for $3 \mathrm{~h}$. The samples were dehydrated in a graded ethanol series $(50 \%, 70 \%, 90 \%, 100 \%)$, infiltrated with propylene oxide and placed into a 70:30 mixture of propylene oxide and resin (Quetol-812; Nissin EM, Tokyo, Japan), after which the propylene oxide volatilised. The samples were then transferred to fresh $100 \%$ resin and polymerised at $60^{\circ} \mathrm{C}$ for $48 \mathrm{~h}$. These blocks were ultra-thin sectioned at $70 \mathrm{~nm}$ using an ultramicrotome, then stained with Lead Stain Solution (18-0875; Sigma-Aldrich). The grids were observed using a transmission electron microscope (JEM-1400Plus; JEOL, Tokyo, Japan) at an acceleration voltage of $80 \mathrm{kV}$. 


\section{RNA interference-mediated gene knockdown}

Transfection of small interfering (si)RNA was performed by electroporation using the Neon Transfection System (Thermo Fisher Scientific). Rat SVF cells suspended in Buffer $\mathrm{R}\left(1.5 \times 10^{5}\right.$ cells per electroporation) were transfected with 50 pmol target-specific Silencer siRNA (Thermo Fisher Scientific), plated onto a 24-well culture plate and cultured in DMEM $(+10 \%$ FBS). Silencer siRNAs used were 4390843 (Scramble), s130429 (Smad1), s131227 (Smad2), s130318 (Smad3), s132211 (Smad4), s133234 (Smad5), s137004 (Smad8), s136654 (Foxo1), s148639 (Foxo3) and s154315 (Foxo4). At $6 \mathrm{~h}$ after transfection, cells were exposed to rosiglitazone and SIS3 for 4 days, followed by treatment with noradrenaline for $2 \mathrm{~h}$. After washing the cells with PBS, cells were used for further analyses.

\section{Plasmids and luciferase gene reporter assay}

The 5' flanking region of the human UCP1 gene (NCBI accession no. NM_021833.4, -4142 to +160 sequence in reference to the transcription start site) was artificially synthesised (GenScript, Piscataway, NJ, USA) and cloned into the pGL3-basic reporter vector (Promega, Madison, WI, USA). The tandem PPREluciferase reporter vector was purchased from Qiagen. The TM-forkhead box $\mathrm{O}$ (FOXO)3 encoding vector (containing a constitutively active mutant human $F O X O 3$ in which all three Akt phosphorylation sites were mutated [triple mutation] to Ala: T32A, S253A, S315A) was artificially generated (Thermo Fisher Scientific). The reporter plasmids were transiently co-transfected with human PPAR $\gamma$, human $P G C l \alpha$ and TM-FOXO3 expression vectors using Lipofectamine 3000 (Thermo Fisher Scientific) into HEK293 cells (ATCC, Manassas, VA, USA; free of mycoplasma contamination). After $24 \mathrm{~h}$ treatment with rosiglitazone, cells were lysed and the reporter activity was measured using the Dual-Luciferase Assay Kit (Promega) and a microplate reader (SH-9000Lab, HITACHI, Tokyo, Japan).

\section{Animal experiments}

All animals were housed under controlled temperatures (23$\left.24^{\circ} \mathrm{C}\right)$ and light-dark cycle $(12 \mathrm{~h})$ conditions, with ad libitum feeding. The $n$ numbers in the animal experiments were set empirically. Each $n$ number is the minimum number of samples for which statistically significant differences can generally be detected. Experimenters were not blind to group assignment and outcome assessment, and no mouse was excluded from the experiments.

Experiment 1: in vivo assessment of treatment in wild-type mice Male, 10-week-old, C57BL/6J Jcl mice (CLEA Japan, Tokyo, Japan) were randomly divided into four groups with similar body weight ( $n=10$ per group), and Alzet osmotic pumps (DURECT, Cupertino, CA, USA) containing rosiglitazone and SIS3 (delivering $5 \mathrm{mg}$ [ $\mathrm{kg}$ body weight ${ }^{-1}$ day $^{-1}$ each); CL316,243 (Sigma-Aldrich) alone (delivering $0.01 \mathrm{mg} \mathrm{kg}^{-1} \mathrm{day}^{-1}$ ); rosiglitazone and SIS3 and CL316,243; or saline $(154 \mathrm{mmol} / \mathrm{l} \mathrm{NaCl})$ were implanted s.c. on the backs of mice under anaesthesia. One week after implantation of the pumps, the mice were dissected and adipose tissues were used for further analyses.

Experiment 2: in vivo assessment of treatment in dietinduced obese mice Male 7-week-old C57BL/6J mice were fed a high-fat diet containing 60\% energy from fat (Research Diets, New Brunswick, NJ, USA) for 10 weeks to induce obesity (diet-induced obese [DIO] mice). The mice were then randomly divided into four groups ( $n=8$ per group) and the rectal temperature of each mouse was measured at 09:00 and 21:00 hours. Alzet osmotic pumps containing rosiglitazone and SIS3 (delivering $5 \mathrm{mg}[\mathrm{kg} \text { body weight }]^{-1}$ day $^{-1}$ each); CL316,243 alone (delivering $0.1 \mathrm{mg} \mathrm{kg}^{-1}$ day $^{-1}$ ); rosiglitazone and SIS3 and CL316,243; or saline were implanted s.c. on the backs of mice under anaesthesia. At 7 days after implantation of the pumps, mice were placed in a chamber for indirect calorimetry and allowed to acclimatise to the surroundings for 3 days. At 10 days, respiratory gas analysis was performed for 2 days as described below. At 13 days, oral glucose-lipid load testing was conducted. The rectal temperature was measured at 21:00 hours (day 12) and 09:00 hours (day 14). At 14 days, blood was collected via the postcaval vein from anaesthetised mice under non-fasting conditions, and each tissue sample was rapidly dissected. All blood analyses were conducted using commercially available kits (ESM Table 3).

\section{Indirect calorimetry}

Energy metabolism studies were performed using ARCO2000 (ARCO System, Chiba, Japan), an MS system for respiratory analysis. $\dot{V} \mathrm{O}_{2}$ and $\dot{V} \mathrm{CO}_{2}$ were measured under ad libitum feeding conditions (high-fat diet) for $48 \mathrm{~h}$ (19:00 hours on day 10 to 19:00 hours on day 12). RQ was calculated from $\dot{V} \mathrm{O}_{2}$ and $\dot{V} \mathrm{CO}_{2}$ values $\left(\mathrm{RQ}=\dot{V} \mathrm{CO}_{2} / \dot{V} \mathrm{O}_{2}\right)$. Oxidation values of lipids and carbohydrates were calculated using the equations of Péronnet and Massicotte [25]. Simultaneously with calorimetry, locomotor activity was also measured using a motion analysis system (Actracer-2000; ARCO System).

\section{Oral glucose-lipid load test}

Glucose-lipid mixed emulsion was prepared by sonicating $10 \%$ corn oil solution containing $10 \%$ glucose, $1 \%$ egg lecithin and $4 \%$ fatty acid-free BSA. Following a $12 \mathrm{~h}$ fast, $10 \mathrm{ml} /$ $\mathrm{kg}$ body weight glucose-lipid emulsion was administered via a gastric tube [26, 27]. Blood samples were obtained from the orbital sinus under anaesthesia with isoflurane at each time 
point, and blood glucose, serum triacylglycerol and NEFA concentrations were measured using a portable blood glucose meter (Accu-Chek Aviva; Roche Diagnostics, Mannheim, Germany) and the kit shown in ESM Table 3.

\section{Statistical analysis}

All measurements are expressed as mean \pm SEM. Comparisons were made using Dunnett's test. Variance analysis was followed by Tukey's test or the Tukey-Kramer method. A $p$ value of less than 0.05 was considered statistically significant.

\section{Results}

\section{Synergistic induction of UCP1 with rosiglitazone, SIS3 and noradrenaline in SVF cells}

In our preliminary experiment to examine the effect of agonists and antagonists of various intracellular signals on Ucpl mRNA expression in SVF cells, we found that the combined treatment of cells with rosiglitazone, SIS3 and noradrenaline synergistically induced Ucp 1 mRNA. Treatment of SVF cells with rosiglitazone and SIS3 for 7 days and noradrenaline for the final $2 \mathrm{~h}$ resulted in a synergistic increase in $U c p 1$ mRNA expression as compared with treatment with each agent alone (Fig. 1a). Use of the selective $\beta 3$ adrenergic receptor agonist CL316,243 instead of noradrenaline also induced Ucp 1 mRNA expression (ESM Fig. 1). To induce synergistic Ucpl expression, treatment with rosiglitazone and SIS3 for 6-10 days followed by noradrenaline stimulation was required (Fig. 1b), with the effect of noradrenaline occurring $2 \mathrm{~h}$ after addition and lasting up to $8 \mathrm{~h}$ (Fig. 1c). Pioglitazone, a PPAR $\gamma$ agonist TZD compound, also showed a synergistic effect similar to that of rosiglitazone; however, a non-TZD partial agonist of PPAR $\gamma$ (nTZDpa) did not show a significant effect (Fig. 1d). RNA interference experiments using specific siRNA for major SMAD family molecules showed that synergistic Ucpl mRNA expression by rosiglitazone and noradrenaline was induced in cells transfected with Smad3 and Smad4 siRNA (Fig. 1e).
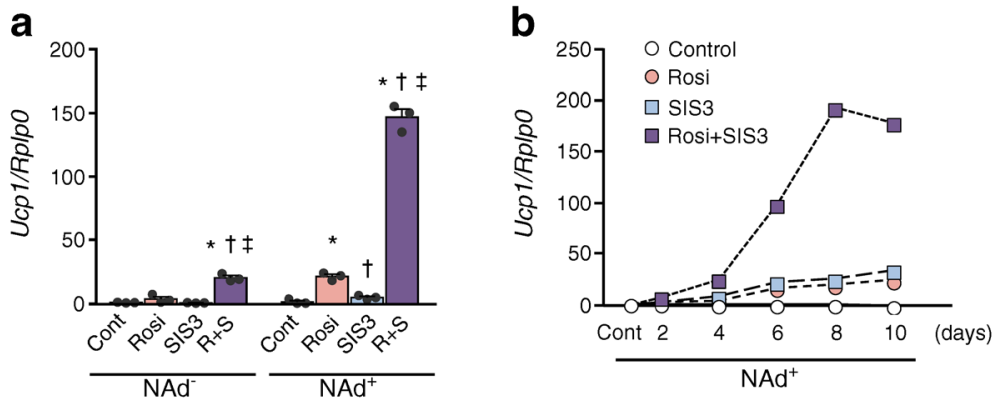
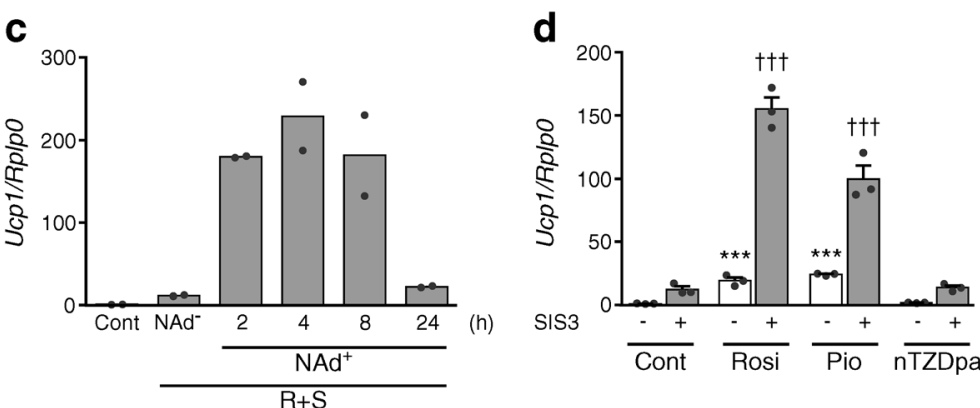

e

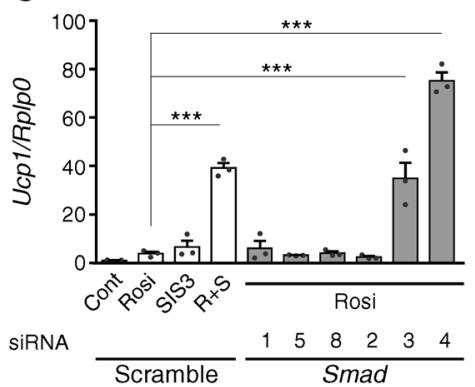

Fig. 1 Synergistic induction of Ucpl with combined rosiglitazone, SIS3 and noradrenaline in SVF cells. (a) Rat SVF cells were untreated (control) or treated with $1 \mu \mathrm{mol} / 1$ rosiglitazone and/or $10 \mu \mathrm{mol} / 1 \mathrm{SIS} 3$ for 7 days; cells were then treated with or without $1 \mu \mathrm{mol} / 1$ noradrenaline for $2 \mathrm{~h}$. ${ }^{*} p<0.05$ vs control in the same NAd treatment group; ${ }^{\dagger} p<0.05$ vs Rosi in the same NAd treatment group; ${ }^{\ddagger} p<0.05$ vs SIS3 in the same NAd treatment group (Tukey's test). (b) SVF cells were untreated (control) or treated with $1 \mu \mathrm{mol} / 1$ rosiglitazone and/or $10 \mu \mathrm{mol} / 1 \mathrm{SIS} 3$ for the indicated time; cells were then treated with $1 \mu \mathrm{mol} / 1$ noradrenaline for $2 \mathrm{~h}$. (c) SVF cells were treated with rosiglitazone + SIS3 for 7 days and with noradrenaline for the indicated time. (d) Comparison between three PPAR $\gamma$ agonists. SVF cells were treated with $1 \mu \mathrm{mol} / 1$ rosiglitazone, pioglitazone or nTZDpa in the presence or absence of SIS3, and then treated with $1 \mu \mathrm{mol} / 1$ noradrenaline for $2 \mathrm{~h}$. $* * * p<0.001 \mathrm{vs}$ control in the SIS3 ${ }^{-}$treatment group; ${ }^{\dagger \dagger} p<0.001$ vs control in the SIS $3^{+}$treatment group (Dunnett's test) (e) SVF cells were transfected with each Smad siRNA and treated with $1 \mu \mathrm{mol} / 1$ rosiglitazone and/or $10 \mu \mathrm{mol} / 1 \mathrm{SIS} 3$ for 4 days, and then with $1 \mu \mathrm{mol} / 1$ noradrenaline for $2 \mathrm{~h}$. The total RNA isolated from whole cell lysate was subjected to real-time RT-PCR. The amount of Ucpl mRNA was normalised to Rplp0 mRNA levels and is expressed as fold change of the corresponding value in the control group (left-hand untreated/Scramble control where there is more than one control). The lines drawn between the bars indicate significant differences between the groups (Dunnett's test, $* * * p<0.001$ ). Values are means \pm SEM of two (b, c) or three (a, d, e) samples. Cont, control; NAd, noradrenaline; Pio, pioglitazone; $\mathrm{R}+\mathrm{S}$, rosiglitazone + SIS3; Rosi, rosiglitazone 


\section{Expression levels of adipocyte marker genes in SVF cells treated with rosiglitazone, SIS3 and noradrenaline}

To uncover changes in cell characteristics upon treatment with the three agents, the expression of various adipocyte marker genes was analysed using real-time PCR. Prdm16 mRNA expression was not increased by any treatment (Fig. 2a), whereas the mRNA expression of PGC1 $\alpha$ (Ppargcla), a cofactor of PPAR $\gamma$, was increased by SIS3 treatment (Fig. 2b). Cidea expression was significantly induced by rosiglitazone and SIS3, and synergistically increased by the combination treatment (Fig. 2c). Expression of Zic1, a classical brown marker, was slightly increased by SIS3 treatment (Fig. 2d), although expression of a similar marker gene, Lhx 8 , did not change (Fig. 2e). In contrast, the expression of Fgf 21 and Cited1, which are specific marker genes of beige adipocytes, increased synergistically with the combined treatment of

Fig. 2 Expression level of adipocyte marker genes in cells treated with rosiglitazone, SIS3 and noradrenaline. Rat SVF cells were untreated (control) or treated with $1 \mu \mathrm{mol} / 1$ rosiglitazone and/or $10 \mu \mathrm{mol} / 1 \mathrm{SIS} 3$ for 7 days; cells were then treated with or without $1 \mu \mathrm{mol} / 1$ noradrenaline for $2 \mathrm{~h}$. mRNAs of brown marker genes $(\mathbf{a}-\mathbf{c})$, classical brown marker genes $(\mathbf{d}, \mathbf{e})$, beige marker genes $(\mathbf{f}-\mathbf{i})$, a white marker gene $(\mathbf{j})$ and adipocyte differentiation marker genes $(\mathbf{k}, \mathbf{l})$ were detected using real-time RT-PCR. The amount of each gene mRNA was normalised to Rplp0 mRNA levels and is expressed as a fold change of the corresponding amount in the untreated control group. Values are means \pm SEM of three samples. Statistical analysis was performed for each set of four groups with or without noradrenaline. $* p<0.05$ vs control in the same NAd treatment group; ${ }^{\dagger} p<0.05$ vs Rosi in the same NAd treatment group; ${ }^{*} p<0.05$ vs SIS 3 in the same NAd treatment group (Tukey's test). Cont, control; NAd, noradrenaline; $\mathrm{R}+\mathrm{S}$, rosiglitazone

+ SIS3; Rosi, rosiglitazone
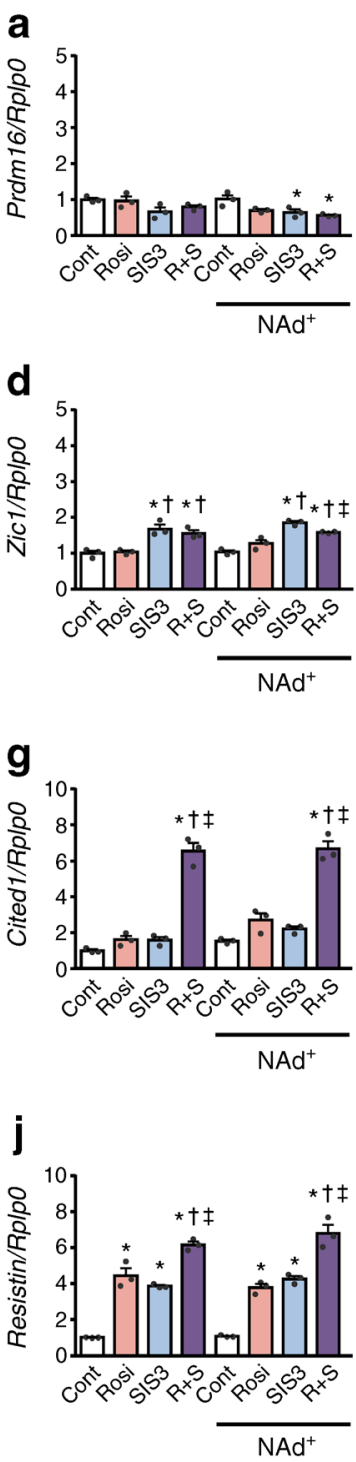

rosiglitazone and SIS3 (Fig. 2f,g). Similarly, the adipocyte differentiation marker genes Fabp4 and Pparg were also significantly increased by the combination treatment of rosiglitazone and SIS3 (Fig. 2k,l).

\section{Decrease in FOXO3 expression by SIS3 induces expression of UCP1 and PPARY target genes in SVF cells}

The FOXO family molecules, comprising transcription factors that regulate cellular metabolism in response to insulin, are known to regulate adipocyte differentiation [28, 29]. Western blot analysis revealed that FOXO1 and FOXO3 levels were markedly decreased in cells treated with SIS3 but not with rosiglitazone (Fig. 3a). Specific siRNA-mediated knockdown of FOXO3 markedly increased Ucpl mRNA (Fig. 3b). Furthermore, Ppargcla, Pparg, Fgf21 and Fabp4 gene expression also increased in FOXO3-knocked down cells on
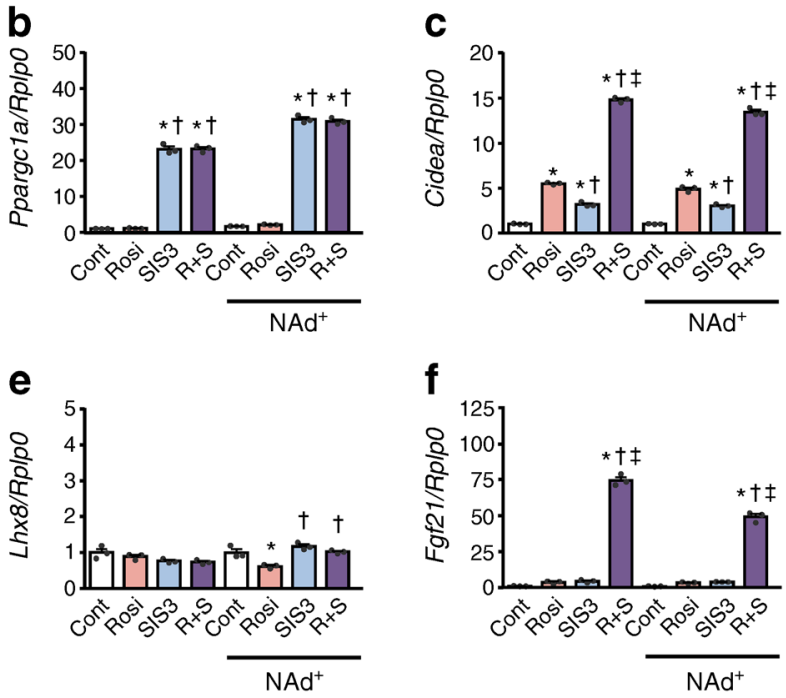

h

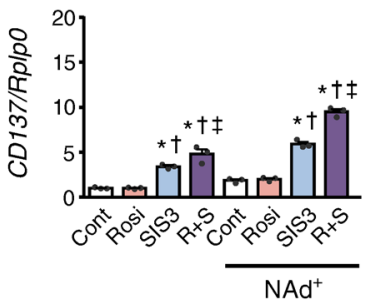

k

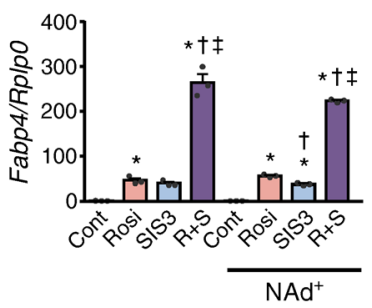

i

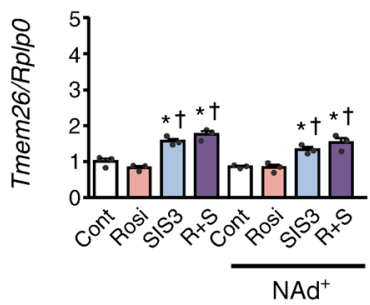

I

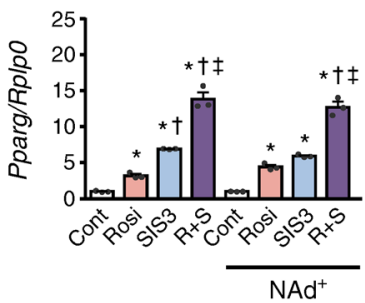



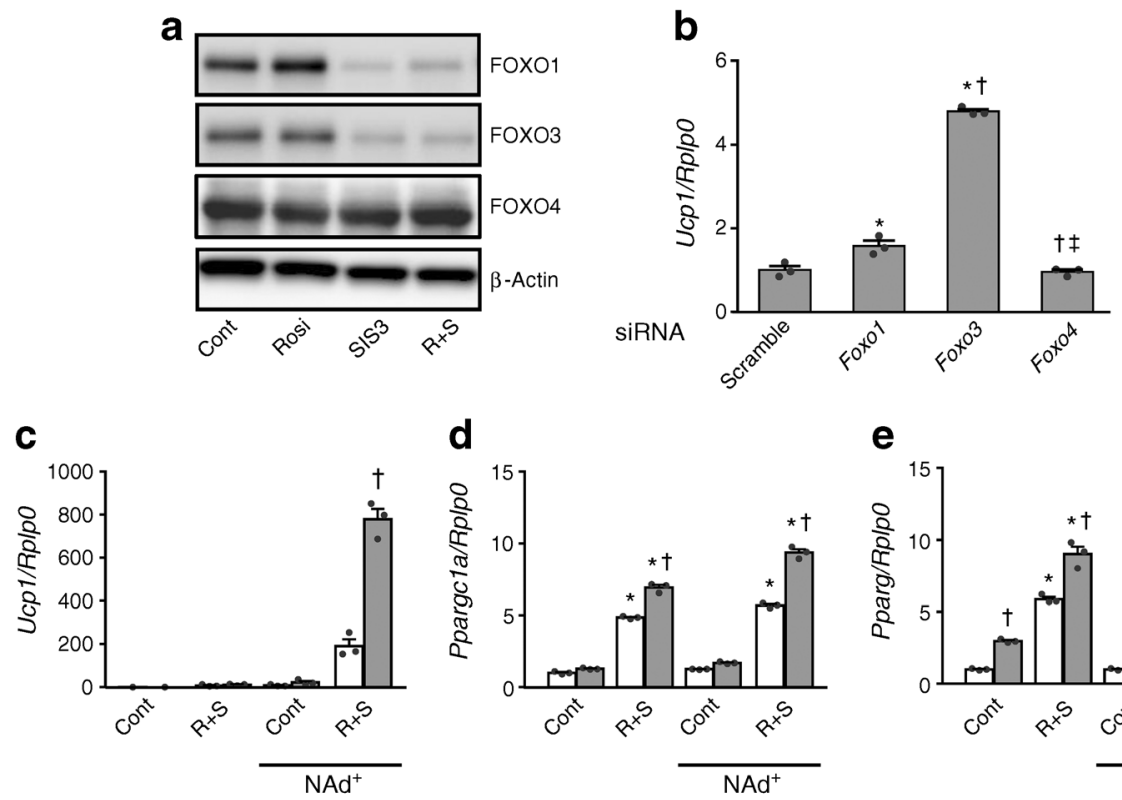

d
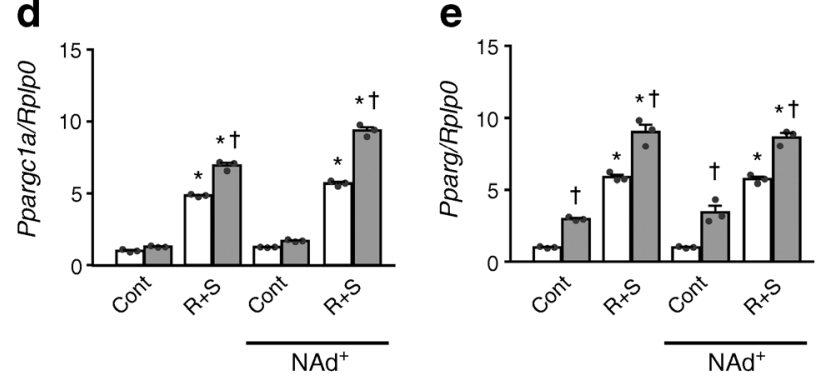

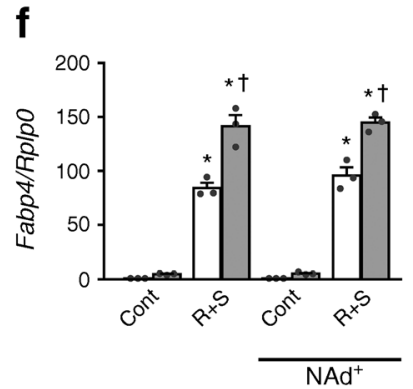

Fig. 3 Effect of FOXO3 suppression on adipocyte gene expression. (a) Rat SVF cells were stimulated with $1 \mu \mathrm{mol} / 1$ rosiglitazone and/or $10 \mu \mathrm{mol} / 1 \mathrm{SIS} 3$ for 7 days. FOXO family protein levels were detected using western blotting. (b) Rat SVF cells were transfected with each Foxo siRNA and treated with $1 \mu \mathrm{mol} / 1$ rosiglitazone and $10 \mu \mathrm{mol} / 1 \mathrm{SIS} 3$ for 4 days, and then with $1 \mu \mathrm{mol} / 1$ noradrenaline for $2 \mathrm{~h}$. The total RNA isolated from whole cell lysate was subjected to real-time RT-PCR. The amount of $U c p 1$ was normalised to Rplp0 mRNA levels and is expressed as a fold change of the corresponding amount in the Scramble group. ${ }^{*} p<0.05$ vs Scramble siRNA transfected group; ${ }^{\dagger} p<0.05$ vs Foxo 1 siRNA transfected group; ${ }^{\star} p<0.05$ vs Foxo 3 siRNA transfected group

combination treatment with rosiglitazone and SIS3 (Fig. 3d-g). Ucp1 gene expression was not detected in two out of three samples in each control-noradrenaline(-) group (Fig. 3c).

\section{Suppression of PPARy/PGC1a-dependent transcriptional activity by $\mathrm{FOXO3}$}

We evaluated the effect of FOXO3 on PPAR $\gamma$-dependent transcriptional activity in HEK293 cells. Treatment of cells cotransfected with PPAR $\gamma$ and PGC1 $\alpha$ expression vectors and a PPRE-luciferase vector with rosiglitazone markedly increased PPAR-dependent luciferase activity (Fig. 4a), whereas this was significantly attenuated by co-expression of a constitutively active FOXO3 generated by triple mutation (TMFOXO3). Similarly, the activity of the human UCP1 promoter was increased by rosiglitazone treatment but suppressed by co-expression of TM-FOXO3 (Fig. 4b).

\section{Induction of UCP1 by a combination of rosiglitazone, SIS3 and CL316,243 in C57BL/6J mice}

Next, we examined the synergistic induction of UCP1 expression by the three-agent combination in in vivo experiments. In these experiments, CL316,243, a synthetic $\beta 3$ adrenergic receptor agonist, was used instead of noradrenaline for pharmacological reasons [30]. An osmotic pump was placed in the back of mice s.c., and rosiglitazone, SIS3 and CL316,243 were chronically administered for 1 week. Expression analyses for each adipose tissue revealed a marked increase in Ucp 1 mRNA and protein expression in inguinal white adipose tissue 


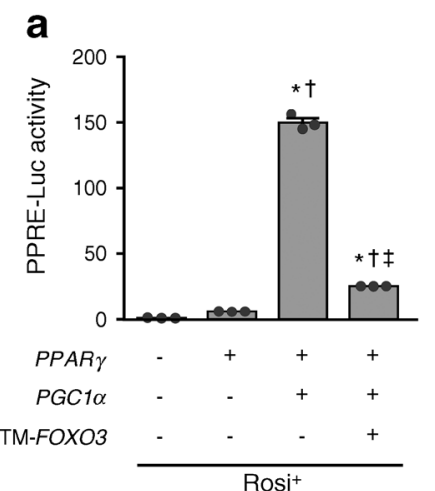

b

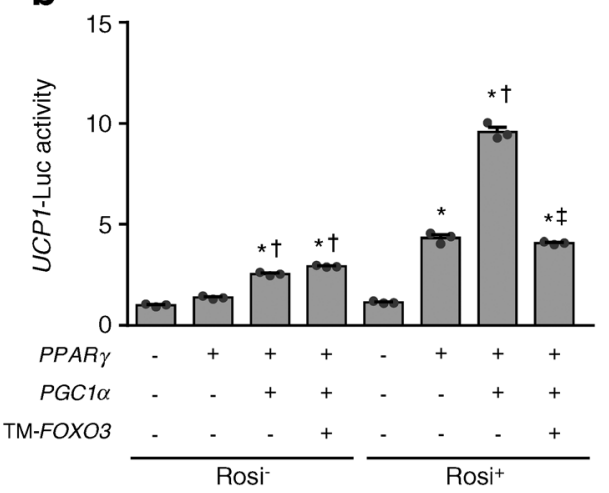

Fig. 4 PPAR $\gamma$ activation induced by rosiglitazone and $\mathrm{PGC} 1 \alpha$ is suppressed by the active form of FOXO3. HEK293 cells were transfected with PPRE (a) or $U C P 1$ promoter (b) reporter plasmids. Concomitantly, $P P A R \gamma, P G C 1 \alpha$ and TM-FOXO3 expression plasmids were cotransfected. The cells were treated with $1 \mu \mathrm{mol} / 1$ rosiglitazone for $24 \mathrm{~h}$, and firefly luciferase activity was measured. Firefly luciferase activity was normalised to Renilla luciferase as relative luciferase activity and is expressed as a fold change of the corresponding amount in the control (no gene transfected, [a] Rosi ${ }^{+},[\mathbf{b}]$ Rosi $^{-}$) group. Values are means \pm SEM of three samples. $* p<0.05$ vs no vector transfected control in the same Rosi treatment group; ${ }^{\dagger} p<0.05$ vs PPAR $\gamma$ transfected in the same Rosi treatment group; ${ }^{\star} p<0.05$ vs PPAR $\gamma$ and $P G C 1 \alpha$ transfected in the same Rosi treatment group (Tukey's test). Luc, luciferase; Rosi, rosiglitazone

(iWAT) with administration of the three agents (Fig. 5a, d, e). Similarly, expression of brown and beige marker genes was increased synergistically with administration of the three agents (ESM Fig. 2). The mRNA expression of Nfe2ll, a newly described thermogenic adaptation regulator, was significantly induced by the three agents (ESM Fig. 3). In transmission electron microscopy analysis of iWAT, miniaturisation of lipid droplets and hyperplasia of mitochondria by the three agents were observed (Fig. 5f).

\section{Effects of combined administration of rosiglitazone, SIS3 and CL316,243 in DIO mice}

We further evaluated the effect of browning of white adipose by the three agents in DIO mice. Chronic administration of the three agents using a subcutaneous osmotic pump for 2 weeks caused synergistic UCP1 expression in obese mice (ESM Fig. 4). At 13 days after starting administration, an oral glucose- lipid tolerance test was performed. The blood glucose level increased markedly $15 \mathrm{~min}$ after oral administration of the emulsion in all groups, but was significantly lower in the all three-agent-treated (combination treatment) group compared with the control group (Fig. 6a) (AUC [mmol/l $\times$ min]: control 1976; rosiglitazone + SIS3 1737; CL316,243 1809; combination treatment 1523). Blood insulin levels were lower in the CL316,243 and combination treatment group than in the control group at 15 and $30 \mathrm{~min}$ after administration (Fig. 6b) (AUC [pmol/l $\times$ min]: control 104,581; rosiglitazone + SIS3 52,081; CL316,243 45,149; combination treatment 44,798; significantly different between control vs CL316,243, control vs combination treatment; Tukey's test, $p<0.05)$. Blood triacylglycerol (Fig. 6c) (AUC [mmol/1 $\times$ min]: control 206.7; rosiglitazone + SIS3 122.0; CL316,243 175.9; combination treatment 118.6; Significantly different control vs rosiglitazone+SIS3, control vs combination treatment group) and NEFA levels (Fig. 6d) (AUC $[\mathrm{mEq} / 1 \times \mathrm{min}]$ : control 126.7; rosiglitazone + SIS3 84.8; CL316,243 109.4; combination treatment 76.3; Significantly different control vs rosiglitazone+SIS3, control vs combination treatment, CL316,243 vs combination treatment group) were lower in the rosiglitazone + SIS3 and combination treatment groups than in the control group.

The body weight of mice at the end of 2 weeks administration was significantly lower in the combination treatment group than control group (Table 1). The body weight of the combination treatment group decreased by $12.3 \%$ compared with before administration. Although it decreased slightly in the rosiglitazone + SIS3 and CL316,243 groups, the difference was not significant in comparison with the control group. The tissue weight of each type of white adipose tissue also decreased in the combination treatment group. In comparison, the weights of BAT, liver and muscle tissues did not change. The core body temperature was significantly higher in the combination treatment group than in the control group during both the day and night. Blood insulin and triacylglycerol levels were significantly lower in the combination treatment group, whereas the adiponectin level increased more than threefold compared with that in the control group.

$\dot{V} \mathrm{O}_{2}$, measured by indirect calorimetry after a 10-day administration of the three agents, showed a non-significant increase of about $14 \%$ and RQ showed a non-significant decrease, compared with the control group (ESM Fig. 5). During this period, no difference was found in the locomotor activity between groups (data not shown).

\section{Discussion}

In the present study, we revealed that the combined treatment of SVF cells with a PPAR $\gamma$ agonist, SMAD3 inhibitor and 


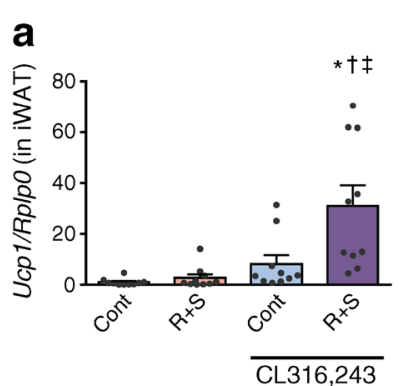

d
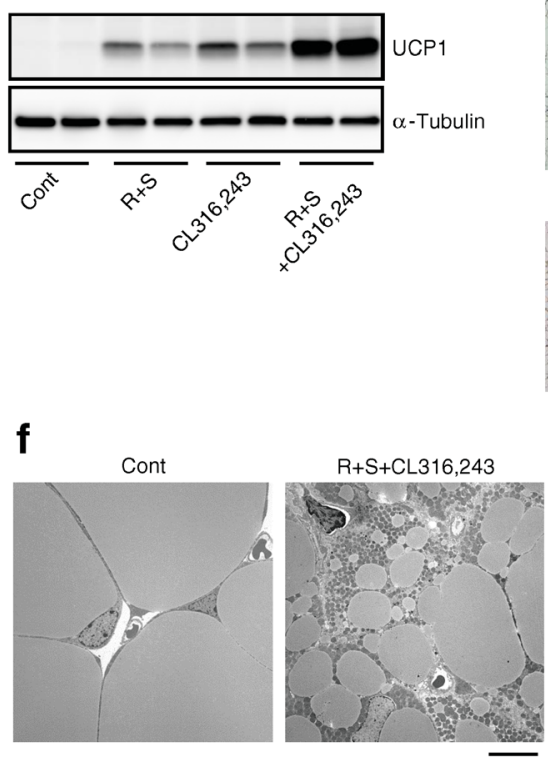

Fig. 5 Induction of UCP1 by a combination of rosiglitazone, SIS3 and CL316,243 in C57BL/6J mice. The mice were administered saline (control) or rosiglitazone and SIS3 (each $5 \mathrm{mg}[\mathrm{kg} \text { body weight }]^{-1}$ day $^{-1}$ ), with or without CL316,243 (0.01 mg [kg body weight $]^{-1}$ day $\left.^{-1}\right)$; all treatments were s.c. using an osmotic pump for 7 days. Total RNA isolated from each adipose tissue, namely iWAT (a), epididymal white adipose tissue (b) and interscapular BAT (c), was subjected to real-time RT-PCR. The amount of Ucp 1 mRNA was normalised to the Rplp 0 mRNA levels and is expressed as fold change of the corresponding amount in iWAT of the control without CL316,243 group. Values are means \pm SEM of nine or ten

adrenergic receptor agonist dramatically induced browning and UCP1 expression, and that these effects were negatively regulated by FOXO3. Furthermore, we have shown that combined administration of the three agents to obese mice effectively increased heat production, reduced body fat and improved glucose tolerance, which accompanied the induction of UCP1 expression in white adipose tissue.

Gene profiling analysis revealed that the expression of beige marker genes, including $F g f 21$ and Citedl, was induced by the three-agent treatment, whereas the expression of classical brown marker genes such as Zicl and Lhx8 did not change, suggesting that the browning of adipose tissue by the three agents likely occurred owing to the induction of beige adipogenesis. Similar to previous results observed for rosiglitazone and noradrenaline treatment [16], treatment with rosiglitazone and SIS3 for at least 4 days, along with subsequent short-term (b, rosiglitazone + SIS3 group) mice $(\mathbf{a}-\mathbf{c}) * p<0.05$ vs control without CL316,243 treatment group; ${ }^{\dagger} p<0.05$ vs R+S without CL316,243 treatment group; ${ }^{\ddagger} p<0.05$ vs control with CL316,243 treatment group (Tukey's test). (d) iWAT lysates were prepared and subjected to western blot analysis using specific antibodies for UCP1 and $\alpha$-tubulin. (e) Representative UCP1 immunostaining in iWAT from each group. Scale bar, $200 \mu \mathrm{m}$. (f) iWAT sections were subjected to transmission electron microscopy. Scale bar, $10 \mu \mathrm{m}$. Cont, control; eWAT, epididymal white adipose tissue; iBAT, interscapular BAT; $\mathrm{R}+\mathrm{S}$, rosiglitazone + SIS3

treatment with noradrenaline, was necessary to obtain the synergistic induction of $U c p 1$ expression by the three agents. In particular, long-term treatment with rosiglitazone increases the cellular amount of PRDM16 by extending the protein's half-life [31], suggesting a contribution of PRDM16 in the three-agentinduced browning and Ucpl expression. Moreover, our results further show that inhibiting SMAD3 signalling in combination with rosiglitazone and noradrenaline is highly effective in inducing browning. SMAD3, which is a target molecule of SIS3, is activated by TGF- $\beta$ and functions as a transcription regulation factor by binding to SMAD-binding elements [32]. Considering the previous finding that SMAD3 suppresses the expression of PGC1 $\alpha$, a coactivator of PPAR $\gamma$, by binding to its promoter region [21], SIS3 treatment may upregulate PGC $1 \alpha$ by inhibiting SMAD3 and thereby increasing PPAR $\gamma$-dependent UCP1 expression. 
Fig. 6 Effects of combined administration of rosiglitazone, SIS3 and CL316,243 on the postprandial response of serum components in obese mice. DIO mice were administered rosiglitazone, SIS3 and/or CL316,243 s.c. using an osmotic pump. At 13 days after administration of the compounds, an oral glucose-lipid load test was conducted. Time-dependent changes in blood glucose (a), serum insulin (b), triacylglycerol (c) and NEFA (d) after glucose-lipid loading were determined. Values are means \pm SEM of eight mice. $* p<0.05$ vs control at each time point (Tukey-Kramer method) a

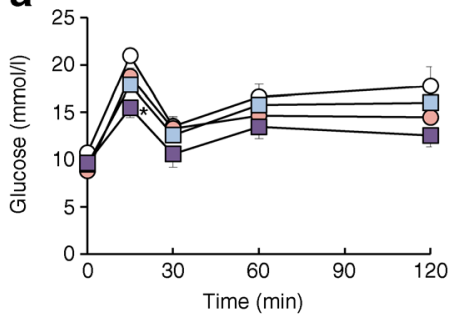

C

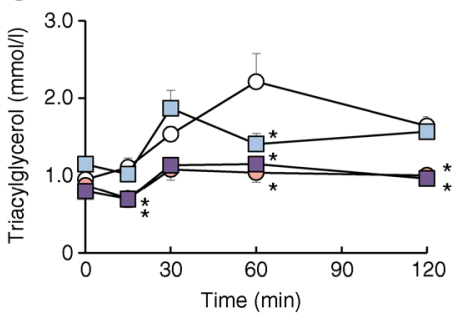

b

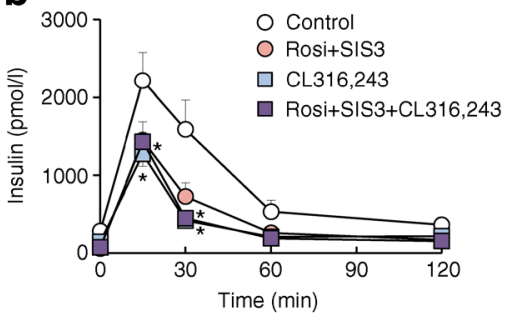

d

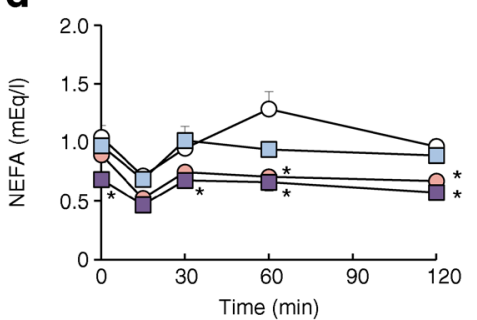

In the present study, multiple lines of evidence suggest that FOXO3 functions as a negative regulator of PPAR $\gamma$ and browning. A predicted mechanism of action is that FOXO3 inhibits the activity of PPAR $\gamma$ by direct protein-protein interaction. In particular, the previous demonstration by coimmunoprecipitation analysis that FOXO1, another member of the FOXO family, directly binds to PPAR $\gamma$ and suppresses the transcriptional activity of PPAR $\gamma$ in adipocytes [33] suggests the possibility that FOXO3 inhibited PPAR $\gamma$ function via a similar mechanism. Notably, however, the inhibition of FOXO3 had a stronger effect on UCP1 expression than did FOXO1 in RNA interference experiments. Although FOXO1 and FOXO3 share sequence motifs and regulate similar target genes [28], each type of FOXO is considered to control diverse biological responses depending on its unique post-translational modifications, cofactor selection and expression level, as indicated by the different phenotype of each FOXO subfamily member-deficient animal model [34, 35], suggesting the possibility that $\mathrm{FOXO} 3$ plays a different role in browning than FOXO1. The protein-protein interaction between FOXO3 and PPAR $\gamma$, as well as the detailed regulation mechanisms of UCP1 expression by the different FOXO proteins, remain to be determined.

Chronic administration of the three agents strongly induced UCP1 expression in mice, especially in subcutaneous adipose tissue. In particular, in the subcutaneous fat of mice administered the three agents, small, multilocular lipid droplets characteristic of BAT were observed, suggesting that the combination treatment induces thermogenic gene expression as well as adipose tissue remodelling. Electron microscopy analysis of the subcutaneous fat showed the presence of numerous developed mitochondria in combination treatment mice. As $\mathrm{PGC} 1 \alpha$ functions as a cofactor of nuclear respiratory factor- $1 / 2$ and induces the expression of mitochondriogenesis-related genes [36], the increased expression of PGC1 $\alpha$ by SMAD3 inhibition might lead to PGC1 $\alpha$-dependent mitochondriogenesis. Alternatively, recent reports indicate that mitophagy, which is a mitochondrial-specific autophagy mechanism, controls brown adipocyte mitochondrial numbers [37]. The findings that $\mathrm{FOXO} 3$ decreases the number of mitochondria by activating mitophagy via PINK1 gene expression [38] and that FOXO3 expression is lower in mitochondrialrich BAT than in white adipose tissue [39] may indicate that FOXO3 contributes to three-agent treatment-mediated browning by regulating the number of mitochondria.

The induction of browning by the three-agent treatment resulted in a significant decrease in body weight and fat mass in obese mice, which was attributable to increased thermogenesis via UCP1 expression and a possible increase in energy expenditure. In addition, administration of the three agents markedly increased serum adiponectin and decreased insulin levels, and improved glucose and lipid tolerance. The increased clearance of blood triacylglycerol by the three agents, which is similar to the results following cold exposure [40], suggests increased activity of beige/brown adipocytes and their possible contribution to the metabolic improvement. Furthermore, increased expression of $\mathrm{Nfe} 2 \mathrm{ll}$, which regulates metabolic and thermogenic function in adaptive thermogenesis [41], may also support the contribution of three-agentinduced browning towards the metabolic improvement in obese mice.

Adiponectin, an adipocytokine secreted from adipose tissue, exhibits many valuable functions including improvement of insulin sensitivity and anti-atherogenic effects [42]. Therefore, the induction of browning by the three agents may be expected to exert an improving and preventive effect towards diabetes, hypertension and cardiovascular diseases by increasing adiponectin production, in addition to the body-fatreducing effect. 
Table 1 Combined administration of rosiglitazone, SIS3 and CL316,243 improves metabolic variables

\begin{tabular}{|c|c|c|c|c|}
\hline Variable & Control & Rosiglitazone + SIS3 & CL316,243 & $\begin{array}{l}\text { Rosiglitazone }+ \\
\text { SIS3 + CL316,243 }\end{array}$ \\
\hline \multicolumn{5}{|l|}{ Body weight (g) } \\
\hline Before admin. & $44.75 \pm 3.30$ & $45.34 \pm 2.83$ & $45.01 \pm 2.43$ & $44.95 \pm 2.61$ \\
\hline After admin. & $44.30 \pm 4.22$ & $43.10 \pm 2.99$ & $41.22 \pm 2.26$ & $39.40 \pm 3.57^{*}$ \\
\hline Change & -0.45 & -2.24 & -3.79 & -5.55 \\
\hline \multicolumn{5}{|l|}{ Tissue weight (mg) } \\
\hline \multicolumn{5}{|l|}{ White adipose tissue } \\
\hline Inguinal & $1780.6 \pm 350.2$ & $1520.0 \pm 117.7$ & $1674.2 \pm 186.3$ & $1414.8 \pm 268.2^{*}$ \\
\hline Interscapular & $425.0 \pm 70.08$ & $362.2 \pm 81.06$ & $238.9 \pm 65.18^{* \dagger}$ & $186.1 \pm 74.06^{* \dagger}$ \\
\hline Epididymal & $2019.8 \pm 292.7$ & $2197.2 \pm 258.8$ & $1771.6 \pm 171.2^{\dagger}$ & $1528.3 \pm 273.0^{* \dagger}$ \\
\hline Perirenal & $319.8 \pm 74.73$ & $277.3 \pm 59.36$ & $232.2 \pm 49.02^{*}$ & $182.8 \pm 63.32^{* \dagger}$ \\
\hline Retroperitoneal & $521.9 \pm 103.2$ & $479.4 \pm 46.29$ & $411.4 \pm 65.99$ & $292.1 \pm 102.3^{* \dagger \dagger}$ \\
\hline BAT & $188.6 \pm 44.68$ & $173.7 \pm 43.58$ & $204.3 \pm 74.42$ & $215.1 \pm 51.41$ \\
\hline Liver & $1672.5 \pm 243.5$ & $1613.3 \pm 158.9$ & $1565.0 \pm 31.09$ & $1670.0 \pm 375.1$ \\
\hline Soleus muscle & $24.90 \pm 4.63$ & $25.07 \pm 1.91$ & $22.28 \pm 3.20$ & $22.03 \pm 2.14$ \\
\hline Gastrocnemius muscle & $332.5 \pm 20.34$ & $319.0 \pm 6.62$ & $312.5 \pm 25.58$ & $315.7 \pm 19.88$ \\
\hline Food intake (g/day) & $3.09 \pm 0.72$ & $2.90 \pm 0.49$ & $3.52 \pm 0.62$ & $3.10 \pm 0.70$ \\
\hline \multicolumn{5}{|l|}{ Rectal temperature $\left({ }^{\circ} \mathrm{C}\right)$} \\
\hline \multicolumn{5}{|l|}{ Day } \\
\hline Before admin. & $36.43 \pm 0.15$ & $36.36 \pm 0.16$ & $36.46 \pm 0.20$ & $36.39 \pm 0.18$ \\
\hline After admin. & $36.48 \pm 0.20$ & $36.84 \pm 0.24$ & $36.80 \pm 0.25$ & $37.20 \pm 0.19^{*}$ \\
\hline \multicolumn{5}{|l|}{ Night } \\
\hline Before admin. & $37.74 \pm 0.20$ & $37.74 \pm 0.18$ & $37.65 \pm 0.27$ & $37.54 \pm 0.13$ \\
\hline After admin. & $37.91 \pm 0.15$ & $38.16 \pm 0.19$ & $37.95 \pm 0.15$ & 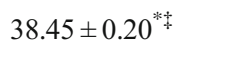 \\
\hline \multicolumn{5}{|c|}{ Biochemical examination of blood } \\
\hline Glucose (mmol/l) & $23.32 \pm 5.92$ & $16.55 \pm 1.15$ & $15.26 \pm 1.09$ & $14.19 \pm 1.51$ \\
\hline Insulin $(\mathrm{pmol} / \mathrm{l})$ & $829.8 \pm 189.6$ & $435.2 \pm 100.7$ & $525.7 \pm 52.1$ & $239.2 \pm 25.4^{*}$ \\
\hline Adiponectin $(\mu \mathrm{g} / \mathrm{ml})$ & $20.79 \pm 0.75$ & $28.74 \pm 4.77$ & $36.85 \pm 6.92$ & $65.56 \pm 15.33^{* \dagger}$ \\
\hline Triacylglycerol (mmol/l) & $1.04 \pm 0.14$ & $0.63 \pm 0.05$ & $0.87 \pm 0.09$ & $0.59 \pm 0.07^{*}$ \\
\hline NEFA (mEq/l) & $0.93 \pm 0.06$ & $0.75 \pm 0.05$ & $0.92 \pm 0.09$ & $0.74 \pm 0.05$ \\
\hline Total cholesterol (mmol/l) & $3.94 \pm 0.37$ & $3.08 \pm 0.13^{*}$ & $3.91 \pm 0.12^{\dagger}$ & $3.68 \pm 0.13$ \\
\hline GOT (IU/l) & $177.63 \pm 51.49$ & $121.18 \pm 29.60$ & $97.23 \pm 10.14$ & $107.46 \pm 9.74$ \\
\hline GPT (IU/1) & $251.71 \pm 73.14$ & $151.70 \pm 31.03$ & $182.62 \pm 40.53$ & $169.90 \pm 25.54$ \\
\hline
\end{tabular}

Data are means \pm SEM of eight mice

DIO mice were administered s.c. rosiglitazone, SIS3 and CL316,243 using an osmotic pump. The adipose tissue of the interscapular region was divided into a brown portion and a white portion according to appearance, and each was weighed

${ }^{*} p<0.05$ vs Control; ${ }^{\dagger} p<0.05$ vs Rosiglitazone+SIS3; ${ }^{\ddagger} p<0.05$ vs CL316,243 (Tukey’s test)

Admin., administration; GOT, glutamic oxaloacetic transaminase; GPT, glutamic pyruvic transaminase

Although we did not observe negative effects in the threeagent group, the safety of systemic SMAD3 inhibition over a long period needs to be scrutinised to fully clarify the efficacy of this method [43].

In summary, we have revealed that the combined regulation of three signals could be useful for inducing UCP1 expression and browning in subcutaneous adipose tissue, thereby leading to reductions in obesity and improvements in various metabolic variables. Furthermore, we identified $\mathrm{FOXO} 3$ as a new regulatory factor in browning and showed that FOXO3 suppresses UCP1 expression by negatively regulating PPAR $\gamma$. Our findings suggest that the synergistic induction of browning by modulating these three signalling pathways may constitute a new and valuable strategy for the treatment of obesity and type 2 diabetes. 
Acknowledgements We thank our colleagues in the Biological Science Laboratories, Kao Corporation, for the helpful discussions.

Data availability The datasets generated during and/or analysed during the current study are available from the corresponding author on reasonable request.

Funding This work was supported financially by Kao Corporation. The study sponsor was not involved in the design of the study; the collection, analysis or interpretation of data; writing the report; or the decision to submit the report for publication.

Duality of interest The authors declare that there is no duality of interest associated with this manuscript.

Author contributions TMu conceived and designed the research. TMa and SK performed experiments. TMu, SK and TMa wrote the manuscript and had final approval of the version to be published. TMu is the guarantor of this work and, as such, had full access to all the data in the study and takes responsibility for the integrity of the data and the accuracy of the data analysis.

\section{References}

1. Bray GA, Bellanger T (2006) Epidemiology, trends, and morbidities of obesity and the metabolic syndrome. Endocrine 29(1):109117. https://doi.org/10.1385/ENDO:29:1:109

2. Cedikova M, Kripnerová M, Dvorakova J, et al (2016) Mitochondria in white, brown, and beige adipocytes. Stem Cells Int 6067349. https://doi.org/10.1155/2016/6067349

3. Klingenberg $M$ (1999) Uncoupling protein - a useful energy dissipator. J Bioenerg Biomembr 31(5):419-430. https://doi.org/ 10.1023/A:1005440221914

4. Cannon B, Nedergaard J (2004) Brown adipose tissue: function and physiological significance. Physiol Rev 84(1):277-359. https://doi. org/10.1152/physrev.00015.2003

5. Feldmann HM, Golozoubova V, Cannon B, Nedergaard J (2009) UCP1 ablation induces obesity and abolishes diet-induced thermogenesis in mice exempt from thermal stress by living at thermoneutrality. Cell Metab 9(2):203-209. https://doi.org/10. 1016/j.cmet.2008.12.014

6. Stanford KI, Middelbeek RJ, Townsend KL et al (2013) Brown adipose tissue regulates glucose homeostasis and insulin sensitivity. J Clin Invest 123(1):215-223. https://doi.org/10.1172/JCI62308

7. Heaton JM (1972) The distribution of brown adipose tissue in the human. J Anat 112(pt 1):35-39

8. Saito M, Okamatsu-Ogura Y, Matsushita M et al (2009) High incidence of metabolically active brown adipose tissue in healthy adult humans: effects of cold exposure and adiposity. Diabetes 58(7): 1526-1531. https://doi.org/10.2337/db09-0530

9. Virtanen KA, Lidell ME, Orava J et al (2009) Functional brown adipose tissue in healthy adults. N Engl J Med 360(15):1518-1525. https://doi.org/10.1056/NEJMoa0808949

10. Yoneshiro T, Aita S, Matsushita M et al (2011) Age-related decrease in cold-activated brown adipose tissue and accumulation of body fat in healthy humans. Obesity (Silver Spring) 19(9):17551760. https://doi.org/10.1038/oby.2011.125

11. Yoneshiro T, Aita S, Matsushita M et al (2011) Brown adipose tissue, whole-body energy expenditure, and thermogenesis in healthy adult men. Obesity (Silver Spring) 19(1):13-16. https:// doi.org/10.1038/oby.2010.105
12. van Marken Lichtenbelt WD, Vanhommerig JW, Smulders NM et al (2009) Cold-activated brown adipose tissue in healthy men. N Engl J Med 360(15):1500-1508. https://doi.org/10.1056/ NEJMoa0808718

13. Wu J, Boström P, Sparks LM et al (2012) Beige adipocytes are a distinct type of thermogenic fat cell in mouse and human. Cell 150(2):366-376. https://doi.org/10.1016/j.cell.2012.05.016

14. Harms M, Seale P (2013) Brown and beige fat: development, function and therapeutic potential. Nat Med 19(10):1252-1263. https:// doi.org/10.1038/nm.3361

15. Sharp LZ, Shinoda K, Ohno H et al (2012) Human BAT possesses molecular signatures that resemble beige/brite cells. PLoS One 7(11):e49452. https://doi.org/10.1371/journal.pone.0049452

16. Petrovic N, Walden TB, Shabalina IG, Timmons JA, Cannon B, Nedergaard J (2010) Chronic peroxisome proliferator-activated receptor $\gamma(\mathrm{PPAR} \gamma)$ activation of epididymally derived white adipocyte cultures reveals a population of thermogenically competent, UCP1-containing adipocytes molecularly distinct from classic brown adipocytes. J Biol Chem 285(10):7153-7164. https://doi. org/10.1074/jbc.M109.053942

17. Rong JX, Qiu Y, Hansen MK et al (2007) Adipose mitochondrial biogenesis is suppressed in $d b / d b$ and high-fat diet-fed mice and improved by rosiglitazone. Diabetes 56(7):1751-1760. https://doi. org/10.2337/db06-1135

18. Kajimura S, Seale P, Tomaru T et al (2008) Regulation of the brown and white fat gene programs through a PRDM16/CtBP transcriptional complex. Genes Dev 22(10):1397-1409. https://doi.org/10. 1101/gad.1666108

19. Yoshida H, Kanamori Y, Asano $\mathrm{H}$ et al (2013) Regulation of brown adipogenesis by the Tgf- $\beta$ family: involvement of Srebp1c in Tgf$\beta$ - and activin-induced inhibition of adipogenesis. Biochim Biophys Acta 1830(11):5027-5035. https://doi.org/10.1016/j. bbagen.2013.06.036

20. Tseng YH, Kokkotou E, Schulz TJ et al (2008) New role of bone morphogenetic protein 7 in brown adipogenesis and energy expenditure. Nature 454(7207):1000-1004. https://doi.org/10.1038/ nature 07221

21. Yadav H, Quijano C, Kamaraju AK et al (2011) Protection from obesity and diabetes by blockade of TGF- $\beta / \mathrm{Smad} 3$ signaling. Cell Metab 14(1):67-79. https://doi.org/10.1016/j.cmet.2011.04.013

22. Park JH, Hur W, Lee SB (2015) Intricate transcriptional networks of classical brown and beige fat cells. Front Endocrinol (Lausanne) 6: 124. https://doi.org/10.3389/fendo.2015.00124

23. Song NJ, Chang SH, Li DY, Villanueva CJ, Park KW (2017) Induction of thermogenic adipocytes: molecular targets and thermogenic small molecules. Exp Mol Med 49(7):e353. https://doi. org/10.1038/emm.2017.70

24. Wilding $\mathrm{J}$ (2006) Thiazolidinediones, insulin resistance and obesity: finding a balance. Int J Clin Pract 60(10):1272-1280. https://doi. org $/ 10.1111 / j .1742-1241.2006 .01128 . x$

25. Péronnet F, Massicotte D (1991) Table of nonprotein respiratory quotient: an update. Can J Sport Sci 16(1):23-29

26. Shimotoyodome A, Fukuoka D, Suzuki J et al (2009) Coingestion of acylglycerols differentially affects glucose-induced insulin secretion via glucose-dependent insulinotropic polypeptide in C57BL/6 $\mathrm{J}$ mice. Endocrinology 150(5):2118-2126. https://doi.org/10.1210/ en.2008-1162

27. Murase T, Yokoi Y, Misawa K et al (2012) Coffee polyphenols modulate whole-body substrate oxidation and suppress postprandial hyperglycaemia, hyperinsulinaemia and hyperlipidaemia. Br J Nutr 107(12):1757-1765. https://doi.org/10.1017/S0007114511005083

28. Onuma H, Vender Kooi BT, Boustead JN, Oeser JK, O’Brien RM (2006) Correlation between FOXO1 (FKHR) and FOXO3a (FKHRL1) binding and the inhibition of basal glucose-6phosphatase catalytic subunit gene transcription by insulin. Mol 
Endocrinol 20(11):2831-2847. https://doi.org/10.1210/me.20060085

29. Munekata K, Sakamoto K (2009) Forkhead transcription factor Foxo1 is essential for adipocyte differentiation. In Vitro Cell Dev Biol Anim 45(10):642-651. https://doi.org/10.1007/s11626-0099230-5

30. MacPherson RE, Castellani L, Beaudoin MS, Wright DC (2014) Evidence for fatty acids mediating CL 316,243-induced reductions in blood glucose in mice. Am J Physiol Endocrinol Metab 307(7): E563-E570. https://doi.org/10.1152/ajpendo.00287.2014

31. Ohno H, Shinoda K, Spiegelman BM, Kajimura S (2012) PPAR $\gamma$ agonists induce a white-to-brown fat conversion through stabilization of PRDM16 protein. Cell Metab 15(3):395-404. https://doi. org/10.1016/j.cmet.2012.01.019

32. Feng XH, Derynck R (2005) Specificity and versatility in TGF- $\beta$ signaling through SMADs. Annu Rev Cell Dev Biol 21(1):659693. https://doi.org/10.1146/annurev.cellbio.21.022404.142018

33. Fan W, Imamura T, Sonoda N et al (2009) FOXO1 transrepresses peroxisome proliferator-activated receptor gamma transactivation, coordinating an insulin-induced feed-forward response in adipocytes. J Biol Chem 284(18):12188-12197. https://doi.org/10. 1074/jbc.M808915200

34. Tzivion G, Dobson M, Ramakrishnan G (2011) FoxO transcription factors; regulation by AKT and 14-3-3 proteins. Biochim Biophys Acta 1813(11):1938-1945. https://doi.org/10.1016/j.bbamcr.2011. 06.002

35. Nakae J, Oki M, Cao Y (2008) The FoxO transcription factors and metabolic regulation. FEBS Lett 582(1):54-67. https://doi.org/10. 1016/j.febslet.2007.11.025
36. Ventura-Clapier R, Garnier A, Veksler V (2008) Transcriptional control of mitochondrial biogenesis: the central role of PGC- $1 \alpha$. Cardiovasc Res 79(2):208-217. https://doi.org/10.1093/cvr/cvn098

37. Altshuler-Keylin S, Shinoda K, Hasegawa Y et al (2016) Beige adipocyte maintenance is regulated by autophagy-induced mitochondrial clearance. Cell Metab 24(3):402-419. https://doi.org/10. 1016/j.cmet.2016.08.002

38. Webb AE, Brunet A (2014) FOXO transcription factors: key regulators of cellular quality control. Trends Biochem Sci 39(4):159169. https://doi.org/10.1016/j.tibs.2014.02.003

39. Furuyama T, Nakazawa T, Nakano I, Mori N (2000) Identification of the differential distribution patterns of mRNAs and consensus binding sequences for mouse DAF-16 homologues. Biochem J 349(pt 2):629-634. https://doi.org/10.1042/bj3490629

40. Bartelt A, Bruns OT, Reimer R et al (2011) Brown adipose tissue activity controls triglyceride clearance. Nat Med 17(2):200-205. https://doi.org/10.1038/nm.2297

41. Bartelt A, Widenmaier SB, Schlein C et al (2018) Brown adipose tissue thermogenic adaptation requires Nrfl-mediated proteasomal activity. Nat Med 24(3):292-303. https://doi.org/10.1038/nm.4481

42. Ruan H, Dong LQ (2016) Adiponectin signaling and function in insulin target tissues. J Mol Cell Biol 8(2):101-109. https://doi.org/ 10.1093/jmcb/mjw014

43. Zhu Y, Richardson JA, Parada LF, Graff JM (1998) Smad3 mutant mice develop metastatic colorectal cancer. Cell 94(6):703-714. https://doi.org/10.1016/S0092-8674(00)81730-4

Publisher's note Springer Nature remains neutral with regard to jurisdictional claims in published maps and institutional affiliations. 